\title{
Kinetostatic analysis of a scissor jack mechanism powered by a tetrad RRR-Ta-RRR
}

\author{
Victor Moise ${ }^{1}$, Ileana Dugaesescu ${ }^{1}$, Lucretia Popa $^{2 *}$, \\ Liviu Ungureanu ${ }^{1}$ and Ştefan-Iulian Moise ${ }^{3}$ \\ ${ }^{1}$ University POLITEHNICA of Bucharest, Romania \\ ${ }^{2}$ INMA Bucharest, 6 Ion Ionescu de la Brad, Bucharest, Romania \\ ${ }^{2}$ Etudes et Productions Schlumberger, Paris, France
}

\begin{abstract}
In a paper previously published by the authors, kinematic analysis of a jack mechanism driven by a motor tetrad was done. For the actual design of the mechanism, the forces and moments acting on the kinematic elements that are part of it must be determined. Also, the driving force in the hydraulic cylinder must be determined, so that the mechanism works in good conditions, for a certain technological task imposed by the design theme. The present paper makes the kinetostatic analysis of the mechanism studied, from the kinematic point of view. Therefore, the reactions in the kinematic couplings of the mechanism, as well as the driving force of the hydraulic cylinder, are determined. In order to determine the reactions of the kinematic couplings, as well as the driving force of the hydraulic cylinder, the masses of the elements, the moments of mechanical inertia, as well as the load force acting on the jack were considered. The results of the calculations were graphically represented, in reaction patterns. In order to verify the correctness of the calculations, the balance force (the hydraulic cylinder motor force) was determined by both the kinetic and the virtual power method, and the results are the same.
\end{abstract}

\section{Introduction}

This mechanism was patented by William P Brown and developed year by year for improving its performances [1]. There are many researches on this mechanism. Manoj R. Patill and S.D. Kachave studied, using Pro-E software, the construction of this mechanism intended to reduce weight of scissor jack and, at the same time, maintaining its strength and service life. The study was made using 3D modelling [2]. This mechanism is also used in the construction of the high scissor lift platform and some research were done using 3D Pro/E software [3]. Other researches about this mechanism were done by researchers from different countries, each of them trying to improve it. [4-11].

Agricultural machines have the most complex mechanisms. Therefore, it is good for the designers to know the methods for determining kinematic and kinetostatic parameters, with the help of calculation procedures on modular groups.

The jack that we studied, as shown in Figure 1, is a special jack, which is very helpful when the agricultural machine breaks down and we have nothing to raise it.

* Corresponding author: Lucreţia Popa, lucretia popa@yahoo.com 
The analysis of the mechanism was made after its synthesis, that is, after determining the dimensions of the kinematic elements. For the synthesis of the mechanism hundreds of equations are written (using the synthesis to approximate a given curve or to guide an element). At such a large number of equations the methods of numerical calculation, the choice of the initial solution of the systems of equations etc. must be mastered.

In order to determine the forces and moments acting on the elements of the mechanism, their positions are considered as known, as well as the distribution of linear and angular velocities and accelerations. At the basis of writing the force equations are used the equations of static balance, the principle of virtual work, the principle of d 'Alembert etc. For each module (structural group) in the structure of the mechanism, the system of balance equations is written, considering the forces and moments applied, the forces and moments of inertia and the forces and moments of reaction. The system of force equations is solved by an appropriate numerical method.

The kinetostatic analysis of the mechanism comprises several stages, namely:

a) kinetostatic study of each modular group;

b) the calculation program for determining the reactions in all the kinematic couplings of the mechanism, as well as the driving force of the active coupling F;

c) tracing the reaction hodographs corresponding to A, D, L and M couplings;

d) drawing the force diagram of the kinematic translational coupling $F$;

e) the table presentation of the reactions in A, B and D couplings for 21 positions of the mechanism elements, as well as of the balance force of the motor torques $\mathrm{F}$, calculated by the kinetostatic method and by the virtual power method.

\section{Material and method}

The kinetostatic analysis of the mechanism was made based on the modular groups of which it is formed. The study of the mechanism as a whole would have led to a large number of equations and implicitly to greater relative and absolute errors of calculation. Regarding the exact calculation, it is known that the calculation errors depend on the numerical method chosen and on the experience of the one who solves the problem.

In this paper, we applied the scientific researches carried out from 1977 to the present. Thus, depending on the evolution of the calculation technique, software libraries were made for the kinematic, kinetostatic and dynamic calculation of the mechanisms in FORTRAN, BASIC, TURBO-PASCAL, C++, MATHCAD, MATLAB etc. [12-23]

These programs have been continuously improved. The calculation program for kinematic, kinetostatic analysis and the animation of the mechanism was made in MATLAB syntax. The program also contains the kinematic analysis of the mechanism, as this is necessary for the kinetostatic analysis.

\section{Results and discussions}

In figure 1, the kinematic diagram of a jack driven mechanism by a motor tetrad can be observed, with the load force acting on the element 9 being highlighted.

To make the kinetostatic analysis it is necessary to know the modules composing the jack mechanism in figure 2 , where $\mathrm{Z}(0)$ is the base, $R R R-T a-R R R(1,2,3,4,5)$ motor tetrad and dyads $\operatorname{RRR}(6,7)$ and $\operatorname{RRR}(8,9)$. For the kinetostatic analysis of the mechanism, the applied and inertia forces in the elements' centroids are reduced; after that, the corresponding functions of each structural group are applied. It is mentioned that the kinetostatic analysis is made in the opposite direction of the kinematic analysis, namely starting with the last structural group kinematically analysed, dyad $R R R(8,9)$ and ending with the motor tetrad $R R R-T a-R R R(1,2,3,4,5)$. 


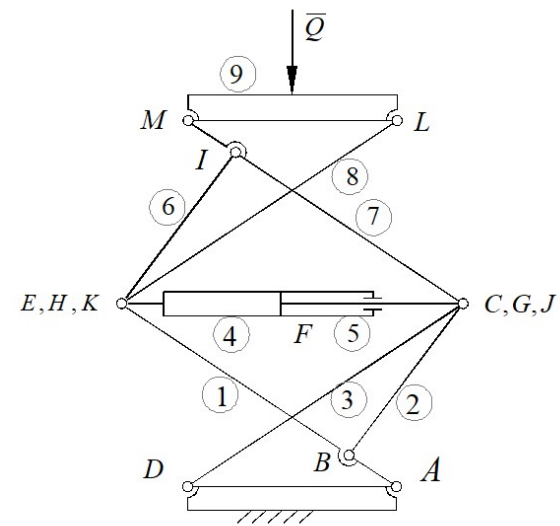

0

Fig. 1. The kinematic diagram of the jack mechanism

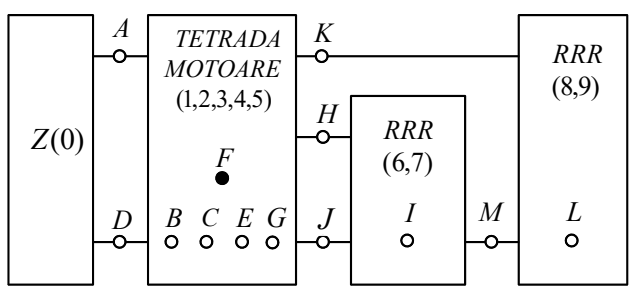

Fig. 2. The multipolar diagram of the jack mechanism

\section{The kinetostatic analysis of the modular groups composing the mechanism Dyad $R R R(8,9)$}

On the dyad $R R R(8,9)$ elements act, according to (Fig. 3,a)

- the load force Q;

- the weight forces: $\bar{G}_{8}=-m_{8} \bar{g}, \bar{G} 9=-m_{9} \bar{g}$;

- the inertia forces: $\bar{F}_{i 8}=-m_{8} \bar{a}_{G 8}, \bar{F}_{i 9}=-m_{9} \bar{a}_{G 9}$, ;

- the resulting moments of the inertia forces: $\bar{M}_{i 8}=-I G_{8} \cdot \bar{\varepsilon}_{8}, \bar{M}_{i 9}=-I G_{9} \cdot \bar{\varepsilon}_{9}$.

The reduction points of the force systems are considered to be in the centroids $G_{8}$ and $G_{9}$ of the kinematic elements 8 and 9.

The accelerations of the reduction points $G_{8}$ and $G_{9}$ being known through the components on the coordinate axes, the inertia forces are:

$$
\bar{F}_{i 8}=F_{i 8 X} \bar{i}+F_{i 8 Y} \bar{j}, \bar{F}_{i 9}=F_{i 9 X} \bar{i}+F_{i 9 Y} \bar{j}, .
$$

The resulting inertia and weight forces are:

$$
\bar{F}_{R 8}=\bar{F}_{8 X}+\bar{F}_{8 Y}, \bar{F}_{R 9}=\bar{F}_{9 X}+\bar{F}_{9 Y},
$$

where:

$$
\begin{aligned}
& \bar{F}_{8 X}=-m_{8} \cdot \bar{a}_{G_{8} X}, \bar{F}_{8 Y}=-m_{8} \cdot\left(\bar{a}_{G_{8} Y}+\bar{g}\right) \\
& \bar{F}_{9 X}=-m_{9} \cdot \bar{a}_{G_{9} X}, \bar{F}_{9 Y}=-m_{9} \cdot\left(\bar{a}_{G_{9} Y}+\bar{g}\right)+\bar{Q} .
\end{aligned}
$$

In relation to the reduction points, the resulting moments are:

$$
\overline{C M}_{8}=-I G_{8} \cdot \bar{\varepsilon}_{8}, \overline{C M}_{7}=-I G_{7} \cdot \bar{\varepsilon}_{7} .
$$

The reactions in the kinematic couplings $K, L$ and $M$ are: $R_{18 X}, R_{18 Y}, R_{98 X}, R_{98 Y}$, $R_{79 X}$ and $R_{79 Y}$. These reactions are the output data of a calculation procedure, drafted by the authors. 


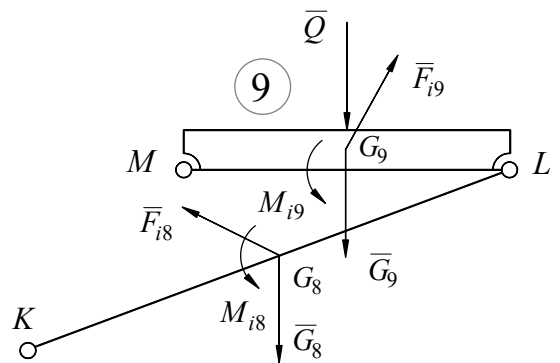

a)

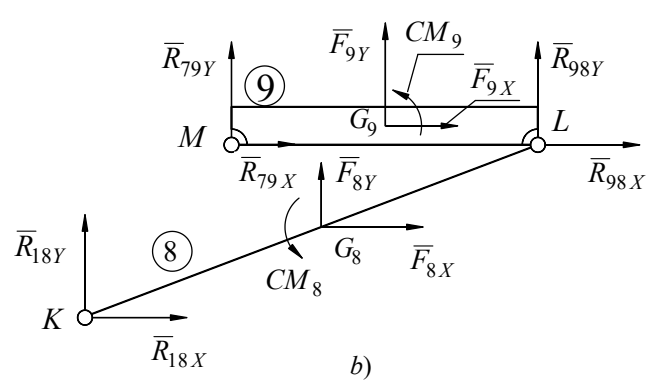

Fig.3. Dyad $R R R(8,9)$

a) highlighting the forces and moments acting on the elements of the structural group;

b) the kinetostatic diagram of dyad $\operatorname{RRR}(8,9)$

\section{Dyad $R R R(6,7)$}

On the elements of dyad $R R R(6,7)$, as shown in (Fig. 4,a) act:

- the reactions of the element 9 on the element 7 of the dyad $R R R(6,7)$ namely: $\bar{R}_{97 X}=-\bar{R}_{79 X}, \bar{R}_{97 Y}=-\bar{R}_{794 Y} ;$

- the weight forces: $\bar{G}_{6}=-m_{6} \bar{g}, \bar{G}_{7}=-m_{7} \bar{g}$;

- the inertia forces: $\bar{F}_{i 6}=-m_{6} \bar{a}_{G_{6}}, \bar{F}_{i 7}=-m_{7} \bar{a}_{G_{7}}$;

- The resulting moments of the inertia forces: $\bar{M} 6=-I G_{6} \cdot \bar{\varepsilon}_{6}, \bar{M}_{i 7}=-I G_{7} \cdot \bar{\varepsilon}_{7}$.

The reduction points of the force systems are considered in the centroids $G_{6}$ and $G_{7}$ of the elements 6 and 7.

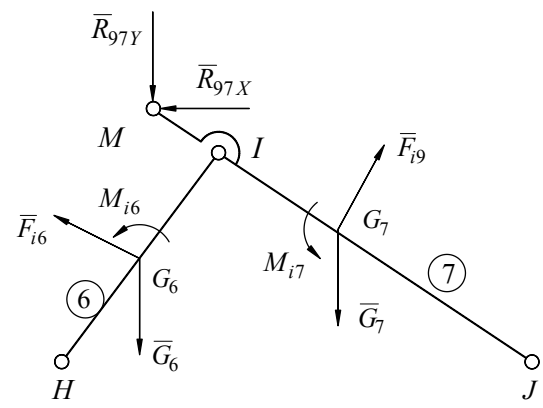

a)

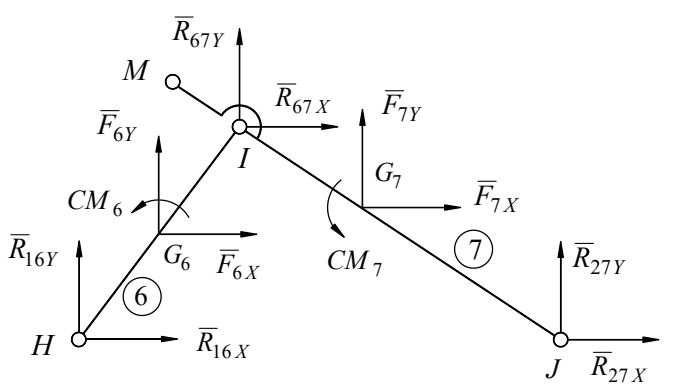

b)

Fig.4. Dyad $R R R(6,7)$

a) highlighting the forces and moments acting on the elements of the structural group;

$b$ ) the kinetostatic diagram of dyad $R R R(6,7)$

The accelerations of the reduction points $G_{6}$ and $G_{7}$ being known through the components on the coordinate axis, the inertia forces are:

$$
\bar{F}_{i 6}=F_{i 6 X} \bar{i}+F_{i 6 Y} \bar{j}, \bar{F}_{i 7}=F_{i 7 X} \bar{i}+F_{i 7 Y} \bar{j} \text {. }
$$

The resulting inertia and weight applied forces, are:

where:

$$
\bar{F}_{R 6}=\bar{F}_{6 X}+\bar{F}_{6 Y}, \bar{F}_{R 7}=\bar{F}_{7 X}+\bar{F}_{7 Y},
$$

$$
\bar{F}_{6 X}=-m_{6} \cdot \bar{a}_{G_{6} X}, \bar{F}_{6 Y}=-m_{6} \cdot\left(\bar{a}_{G_{6} Y}+\bar{g}\right)
$$




$$
\bar{F}_{7 X}=-m_{7} \cdot \bar{a}_{G_{7} X}+\bar{R}_{97 X}, \bar{F}_{7 Y}=-m_{7} \cdot\left(\bar{a}_{G_{7} Y}+\bar{g}\right)+\bar{R}_{97 Y} .
$$

In relation to the reduction points, the resulting moments are:

$$
\overline{C M}_{6}=-I G_{6} \cdot \bar{\varepsilon}_{6}, \overline{C M}_{7}=-I G_{7} \cdot \bar{\varepsilon}_{7}+\overline{G_{7} M} \times\left(\bar{R}_{97 X}+\bar{R}_{97 Y}\right) .
$$

The reactions in the kinematic couplings $H, I$ and $J$ are: $R_{16 X}, R_{16 Y}, R_{67 X}, R_{67 Y}$, $R_{27 X}$ and $R_{27 Y}$. These reactions are the output data of a calculation procedure.

\section{The motor tetrad $R R R-T a-R R R(1,2,3,4,5)$}

On the elements of the motor tetrad $R R R-T a-R R R(1,2,3,4,5)$ act (Fig.5):

- The reactions of the elements $\mathbf{6}$ and $\mathbf{8}$ on the element $\mathbf{1}$ of the motor tetrad $R R R-T a$ $R R R(1,2,3,4,5)$, are: $\bar{R}_{61 X}=-\bar{R}_{16 X}, \bar{R}_{61 Y}=-\bar{R}_{16 Y}, \bar{R}_{81 X}=-\bar{R}_{18 X}, \bar{R}_{81 Y}=-\bar{R}_{18 Y}$.

- the reactions of the element 7 on the element 2 of the motor tetrad RRR-Ta$R R R(1,2,3,4,5)$, are: $\bar{R}_{72 X}=-\bar{R}_{27 X}, \bar{R}_{72 Y}=-\bar{R}_{27 Y}$

- the weight forces: $\bar{G}_{1}=-m_{1} \bar{g}, \bar{G}_{2}=-m_{2} \bar{g}, \bar{G}_{3}=-m_{3} \bar{g}, \bar{G}_{4}=-m_{4} \bar{g}$, $\bar{G}_{5}=-m_{5} \bar{g}$

- the inertia forces: $\quad \bar{F}_{i 1}=-m_{1} \bar{a}_{G 1}, \quad \bar{F}_{i 2}=-m_{2} \bar{a}_{G 2}, \quad \bar{F}_{i 3}=-m_{3} \bar{a}_{G 3}$, $\bar{F}_{i 4}=-m_{4} \bar{a}_{G 4}, \bar{F}_{i 5}=-m_{5} \bar{a}_{G 5} ;$

- the resulting moments of the inertia forces: $\bar{M}_{i 1}=-I G_{1} \cdot \bar{\varepsilon}_{1}, \bar{M}_{i 2}=-I G_{2} \cdot \bar{\varepsilon}_{2}$, $\bar{M}_{i 3}=-I G_{3} \cdot \bar{\varepsilon}_{3}, \bar{M}_{i 4}=-I G_{4} \cdot \bar{\varepsilon}_{4}, \bar{M}_{i 5}=-I G_{5} \cdot \bar{\varepsilon}_{5}\left(\bar{\varepsilon}_{4}=\bar{\varepsilon}_{5}\right)$.

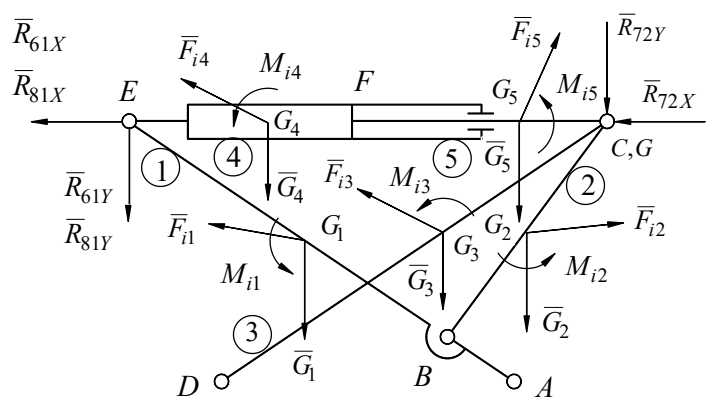

Fig. 5. The motor tetrad $R R R-T a-R R R(1,2,3,4,5)$. Highlighting the forces and moments acting on the elements of the structural group

The reduction points of the forces systems are considered in the centroids $G_{1}, G_{2}, G_{3}$ $G_{4}$ and $G_{5}$, corresponding to the elements $\mathbf{1}, \mathbf{2}, \mathbf{3}, \mathbf{4}$ and $\mathbf{5}$. The kinematic parameters of the points $G_{1}, G_{2}, G_{3} G_{4}$ and $G_{5}$ are calculated using a calculation procedure drafted by the authors. The resulting inertia, weight and reaction forces are as follows (Fig.6).

$\bar{F}_{R 1}=\bar{F}_{1 X}+\bar{F}_{1 Y}, \bar{F}_{R 2}=\bar{F}_{2 X}+\bar{F}_{2 Y}, \bar{F}_{R 3}=\bar{F}_{3 X}+\bar{F}_{34 Y}$,

$\bar{F}_{R 4}=\bar{F}_{4 X}+\bar{F}_{4 Y}, \bar{F}_{R 5}=\bar{F}_{5 X}+\bar{F}_{5 Y}$,

where:

$$
\begin{aligned}
& \bar{F}_{1 X}=-m_{1} \cdot \bar{a}_{G 1 X}+\bar{R}_{61 X}+\bar{R}_{81 X} ; \bar{F}_{1 Y}=-m_{1} \cdot\left(\bar{a}_{G 1 Y}+\bar{g}\right)+\bar{R}_{61 Y}+\bar{R}_{81 Y} \\
& \bar{F}_{2 X}=-m_{2} \cdot \bar{a}_{G_{2} X}+\bar{R}_{72 X} ; \bar{F}_{2 Y}=-m_{2} \cdot\left(\bar{a}_{G_{2} Y}+\bar{g}\right)+\bar{R}_{72 Y} \\
& \bar{F}_{3 X}=-m_{6} \cdot \bar{a}_{G 3 X} ; \bar{F}_{3 Y}=-m_{3} \cdot\left(\bar{a}_{G 3 Y}+\bar{g}\right) \\
& \bar{F}_{4 X}=-m_{4} \cdot \bar{a}_{G 4 X} ; \bar{F}_{4 Y}=-m_{4} \cdot\left(\bar{a}_{G 4 Y}+\bar{g}\right)
\end{aligned}
$$




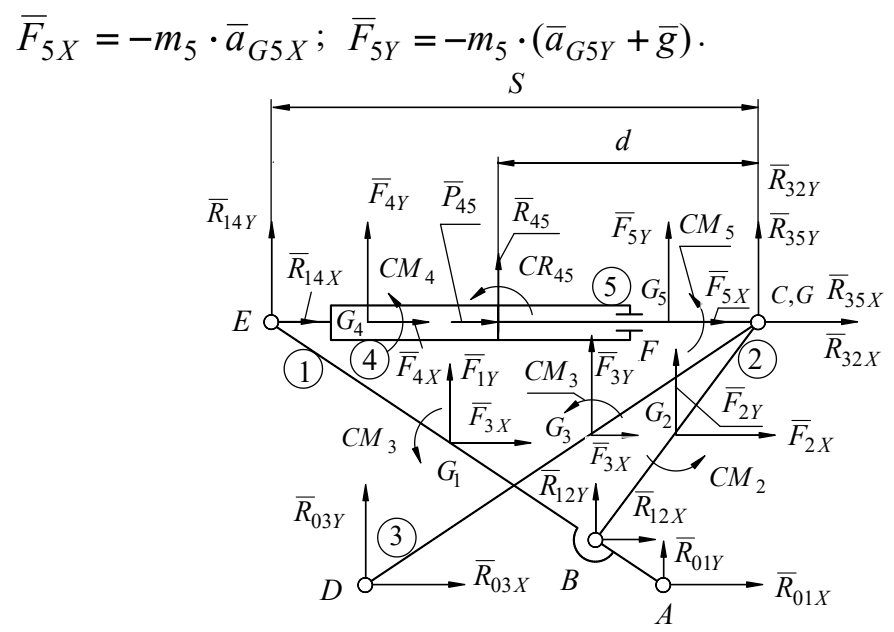

Fig.6. The motor tetrad $R R R-T a-R R R(1,2,3,4,5)$.

The kinetostatic diagram of the motor tetrad $R R R-T a-R R R(1,2,3,4,5)$

In relation to the reduction points, the resulting moments are:

$$
\begin{aligned}
& \overline{C M}_{1}=-I G_{1} \cdot \bar{\varepsilon}_{1}+\bar{G}_{1} E \times\left(\bar{R}_{61 X}+\bar{R}_{61 Y}\right)+\overline{G_{1} E} \times\left(\bar{R}_{61 X}+\bar{R}_{81 Y}\right) ; \\
& \overline{C M}_{2}=-I G_{2} \cdot \bar{\varepsilon}_{2}+\bar{G}_{2} C \times\left(\bar{R}_{72 X}+\bar{R}_{72 Y}\right) ; \\
& \overline{C M}_{3}=-I G_{3} \cdot \bar{\varepsilon}_{3} ; \overline{C M}_{4}=-I G_{4} \cdot \bar{\varepsilon}_{4} ; \overline{C M}_{5}=-I G_{5} \cdot \bar{\varepsilon}_{5}\left(\bar{\varepsilon}_{4}=\bar{\varepsilon}_{5} .\right.
\end{aligned}
$$

The reactions in the kinematic couplings $A, B, C, D, E, F$ and $G$ are: $R_{01 X}, R_{01 Y}, R_{12 X}$ , $R_{12 Y}, R_{32 X}, R_{32 Y}, R_{03 X}, R_{03 Y}, R_{14 X}, R_{14 Y}, R_{45}, C R_{45}, R_{35 X}$ and $R_{35 Y}$. The driving force in the hydraulic cylinder is $P_{45}$. These sizes are the output data of a calculation procedure drafted by the authors.

To determine the kinematic and kinetostatic parameters of the mechanism elements, the following data were considered:

- $\quad$ the elements dimensions: $A B=0.061 \mathrm{~m}, A E=0.280 \mathrm{~m}, B C=0.1598 \mathrm{~m}$;

$$
\begin{aligned}
& C D=0.280 \mathrm{~m}, \quad B E=A E-A B, J M=0.280 \mathrm{~m}, J I=B E, H I=B C, K L=A E, \\
& M L=0.180 \mathrm{~m} ;
\end{aligned}
$$

- $\quad$ the positions of the couplings adjacent to the base: $X A=0.0 \mathrm{~m}, Y A=0.0 \mathrm{~m}, X D$ $=-0.180 \mathrm{~m}, Y D=0.0 \mathrm{~m}$;

- $\quad$ the initial length of the hydraulic cylinder: $S O=0.270 \mathrm{~m}$;

- $\quad$ the hydraulic cylinder piston stroke: $=0.098 \mathrm{~m}$;

- $\quad$ the relative speed of the piston to the cylinder: $d S / d t=-0.025 \mathrm{~m} \cdot \mathrm{s}^{-1}$;

- the relative acceleration of the piston to the cylinder is considered equal to zero

- $\quad$ the mass of the kinematic elements: $m_{1}=1.5 \mathrm{~kg}, m_{2}=1.0 \mathrm{~kg}, m_{3}=1.5 \mathrm{~kg}$, $m_{4}=1.5 \mathrm{~kg}, m_{5}=1.0 \mathrm{~kg}, \mathrm{~m}_{6}=1.0 \mathrm{~kg}, \mathrm{~m}_{7}=1.5 \mathrm{~kg}, \mathrm{~m}_{8}=1.5 \mathrm{~kg}, \mathrm{~m}_{9}=1.0 \mathrm{~kg}$;

- $\quad$ the mechanical inertia moments: $I G 1=1.5 \mathrm{kgm}^{2}, I G 2=1.0 \mathrm{kgm}^{2}, I G 3=1.5 \mathrm{kgm}^{2}$, $I G 4=1.5 \mathrm{kgm}^{2}, I G 5=1.0 \mathrm{kgm}^{2}, I G 6=1.0 \mathrm{kgm}^{2}, I G 7=1.5 \mathrm{kgm}^{2}, I G 8=1.5 \mathrm{kgm}^{2}, I G 9=1.0$ $\mathrm{kgm}^{2}$

- $\quad$ the load force: $Q=2000 \mathrm{~N}$.

Using the calculation program made by MATLAB syntax, the values of reactions in the kinematic couplings of the mechanism are obtained, as well as the driving force in the hydraulic cylinder.

In figures 7, 8, 9 and 10, the hodographs of reactions in the kinematic couplings $A, D$, $L$ and $M$ are shown. 


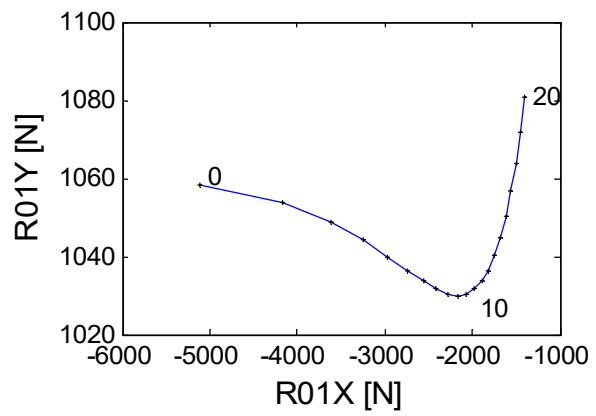

Fig. 7. The hodograph of reactions in coupling A

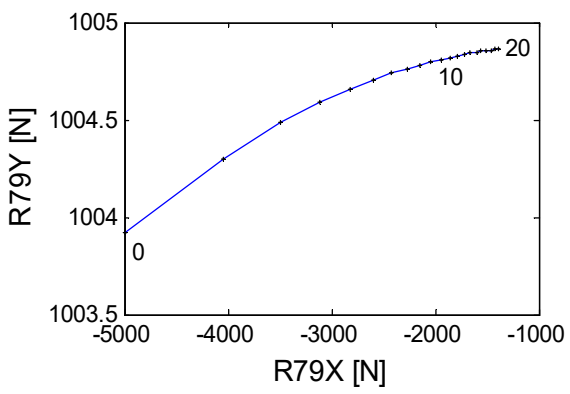

Fig. 9. The hodograph of reactions in coupling $\mathrm{L}$

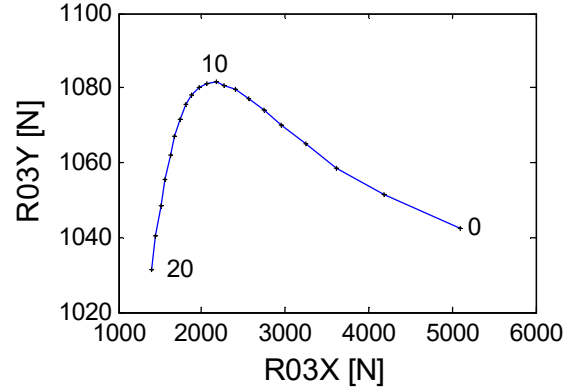

Fig. 8. The hodograph of reactions in coupling D

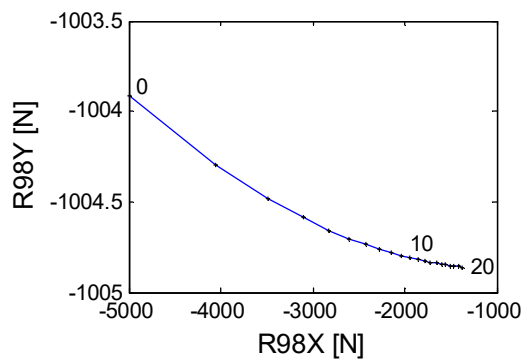

Fig. 10. The hodograph of reactions in coupling $\mathrm{M}$

In Fig. 11, the diagram of the driving force in the hydraulic cylinder is presented.

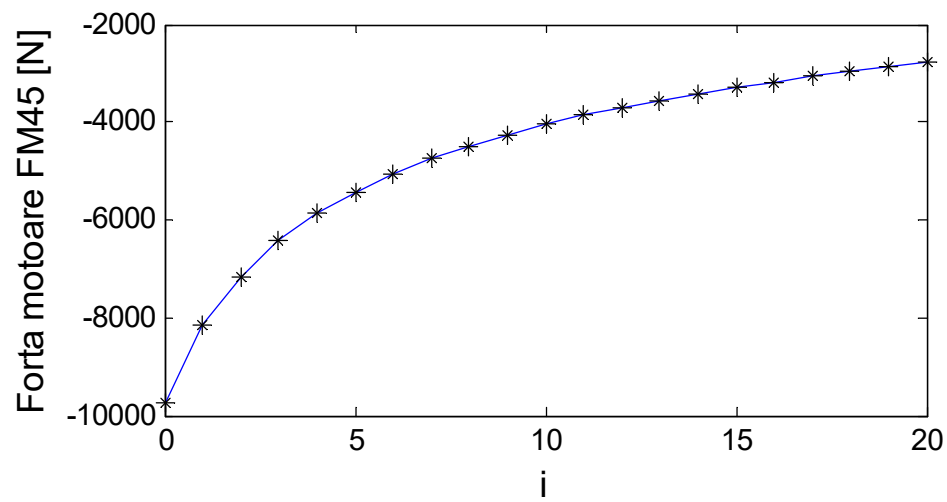

Fig. 11. The diagram of the driving force in the hydraulic cylinder 4-5

In the calculation procedure, the driving force was considered to be positive when the hydraulic cylinder is relaxing, that is when the piston rod is getting out of the cylinder.

In table 1, the components' values of the reactions in $A, D$ and $B$ couplings are presented. 
Table 1 Reactions in $A, D$ and $B$ couplings

\begin{tabular}{|c|c|c|c|c|c|c|}
\hline Pos. & R01X & R01Y & R03X & R03Y & R12X & R12Y \\
\hline 0 & -5108.92 & 1058.11 & 5109.33 & 1042.36 & -176.47 & -44.19 \\
\hline 1 & -4179.34 & 1053.83 & 4179.58 & 1051.36 & -30.55 & -5.98 \\
\hline 2 & -3624.83 & 1048.83 & 3625.04 & 1058.70 & 9.01 & 8.53 \\
\hline 3 & -3244.66 & 1044.08 & 3244.86 & 1064.79 & 18.27 & 13.48 \\
\hline 4 & -2962.06 & 1039.90 & 2962.25 & 1069.82 & 17.18 & 13.92 \\
\hline 5 & -2740.42 & 1036.39 & 2740.60 & 1073.91 & 12.52 & 12.13 \\
\hline 6 & -2559.77 & 1033.62 & 2559.96 & 1077.09 & 6.99 & 9.28 \\
\hline 7 & -2408.21 & 1031.61 & 2408.40 & 1079.41 & 1.72 & 6.04 \\
\hline 8 & -2278.10 & 1030.39 & 2278.29 & 1080.88 & -2.80 & 2.82 \\
\hline 9 & -2164.34 & 1029.96 & 2164.53 & 1081.49 & -6.37 & -0.10 \\
\hline 10 & -2063.32 & 1030.35 & 2063.51 & 1081.25 & -8.94 & -2.54 \\
\hline 11 & -1972.47 & 1031.56 & 1972.66 & 1080.16 & -10.51 & -4.36 \\
\hline 12 & -1889.84 & 1033.60 & 1890.04 & 1078.23 & -11.12 & -5.44 \\
\hline 13 & -1813.99 & 1036.48 & 1814.18 & 1075.43 & -10.84 & -5.72 \\
\hline 14 & -1743.76 & 1040.21 & 1743.95 & 1071.78 & -9.72 & -5.12 \\
\hline 15 & -1678.27 & 1044.79 & 1678.46 & 1067.26 & -7.83 & -3.58 \\
\hline 16 & -1616.79 & 1050.24 & 1616.99 & 1061.87 & -5.24 & -1.05 \\
\hline 17 & -1558.75 & 1056.55 & 1558.95 & 1055.61 & -2.00 & 2.52 \\
\hline 18 & -1503.66 & 1063.74 & 1503.86 & 1048.46 & 1.83 & 7.16 \\
\hline 19 & -1451.13 & 1071.82 & 1451.33 & 1040.42 & 6.20 & 12.93 \\
\hline 20 & -1400.83 & 1080.79 & 1401.03 & 1031.48 & 11.04 & 19.87 \\
\hline
\end{tabular}

In table 2, the values of the driving force in the hydraulic cylinder are presented, values calculated through the kinetostatic method and the virtual power method.

The small differences are due to the calculation errors.

Table 2 Driving force in the hydraulic cylinder

\begin{tabular}{|c|c|c|c|c|c|}
\hline Pos. & P45_CINET & FE_PV45 & Pos. & P45_CINET & FE_PV45 \\
\hline $\mathbf{0}$ & -9723.68 & -9723.05 & & & \\
\hline $\mathbf{1}$ & -8175.58 & -8175.30 & $\mathbf{1 1}$ & -3861.88 & -3861.86 \\
\hline $\mathbf{2}$ & -7159.70 & -7159.55 & $\mathbf{1 2}$ & -3697.68 & -3697.67 \\
\hline $\mathbf{3}$ & -6428.33 & -6428.24 & $\mathbf{1 3}$ & -3548.70 & -3548.69 \\
\hline $\mathbf{4}$ & -5868.95 & -5868.88 & $\mathbf{1 4}$ & -3412.60 & -3412.59 \\
\hline $\mathbf{5}$ & -5422.68 & -5422.63 & $\mathbf{1 5}$ & -3287.52 & -3287.51 \\
\hline $\mathbf{6}$ & -5055.45 & -5055.42 & $\mathbf{1 6}$ & -3171.93 & -3171.92 \\
\hline $\mathbf{7}$ & -4746.01 & -4745.98 & $\mathbf{1 7}$ & -3064.59 & -3064.59 \\
\hline $\mathbf{8}$ & -4480.32 & -4480.30 & $\mathbf{1 8}$ & -2964.48 & -2964.48 \\
\hline $\mathbf{9}$ & -4248.72 & -4248.70 & $\mathbf{1 9}$ & -2870.73 & -2870.73 \\
\hline $\mathbf{1 0}$ & -4044.27 & -4044.25 & $\mathbf{2 0}$ & -2782.62 & -2782.61 \\
\hline
\end{tabular}

\section{Conclusions}

Obtaining the values of reactions in the kinematic couplings of the mechanism allows passing to the next step, namely the actual design of the kinematic elements. To check the calculations, the driving force in the hydraulic cylinder 4-5 was determined also through the virtual power method and the results were identical to those in the kinetostatic calculations. Depending on the driving force of the hydraulic cylinder, as well as on the general dimensions, the hydraulic cylinder will be chosen from a provider of hydraulic elements.

This paper was financed by grant of the Romanian Education and Research Ministry, through Programme 1 - Development of the national research-development system, sub-programme 1.2 Institutional performance - Projects for financing excellence in RDI, contract no. 16PFE. 


\section{References}

1. W.P. Brown, Scissors Jack. US Patent Office (1946).

2. Manoj R., Patil, S.D. Kachave, Int. J. Mech. Eng. \& Rob. Res., 4(1), (2015).

3. Tian Hongyu, Zhang Ziyi, Design and Simulation Based on Pro/E for a Hydraulic Lift Platform in Scissors Type, Procedia Engineering, 16, 772-781, (2011).

4. Islam Md Toufiqul, Yin Cheng, Jian Shengqi, Rolland Luc, Dynamic analysis of Scissor Lift mechanism through bond graph modelling, IEEE/ASME International Conference on Advanced Intelligent Mechatronics, AIM, (2016).

5. Yuantao Sun, Sanmin Wang, James K. Mills \& Changjian Zhi, Kinematics and dynamics of deployable structures with scissor-like-elements based on screw theory, Chinese Journal of Mechanical Engineering, 27, 655-662, (2014).

6. J. Hartsell, The development of a dynamic scissor lift model, West Virginia University. The Research Repository. (2010).

7. Langbecker, T. (1999). Kinematic Analysis of Deployable Scissor Structure. International Journal of Space Structures, 14(1), 1-15.

8. M. Bariskan, Final Project_Design and FEM Analysis of Scissor Jack https://www.slideshare.net/MehmetBariskan/final-project-design-and-femanalysis-of-scissor-jack, (2014).

9. https://www.engineersedge.com/mechanics_machines/scissor-lift.htm

10. C.S. Dhamak, D.S. Bajaj, V.S. Aher, Design and Optimization of Scissor Jack, IJAPME, 2(1), 29-34, (2016).

I. Dugaesescu, L. Popa, Şt. Moise, V. Moise, V. Ştefan, Al. Rotaru. ERDev, (2019).

11. I.I. Artobolevski, Theorie des mecanismes et des machines. (Theory of Mechanisms and Machines), (1977).

12. B. Demidovich, A.I. Maron, Éléments de calcul numérique, (1987).

13. N. Manolescu, Fr. Kovacs, A. Oranescu, Teoria mecanismelor şi a maşinilor (The Theory of Mechanisms and Machines), (1972).

14. V. Moise, Cinematica şi controlul mecanismelor manipulatoare cu 4 grade de mobilitate, $\mathrm{PhD}$ Thesis, University Politehnica of Bucharest, (1986).

15. V. Moise, I. Simionescu, M. Ene, M. Neacşa, I.A. Tabara, Analiza mecanismelor aplicate (Analysis of Applied Mechanisms), (2007).

16. V. Moise, I. Simionescu, M. Ene, Al. Rotaru, Analysis of planar mechanisms with articulated bars. Applications in MATLAB (Analiza mecanismelor plane cu bare articulate. Aplicații în MATLAB). (2015).

17. V. Moise, E. Maican, Şt Moise, Numerical methods. Applications in MATLAB (Metode numerice. Aplicații în MATLAB). (2016).

18. Chr. Pelecudi, Precizia mecanismelor (The precision of the mechanisms). (1975).

19. Chr. Pelecudi, D. Maros, V. Merticaru, N. Pandrea, I. Simionescu, Mecanisme (Mechanisms), (1985).

I. $\quad$ Simionescu, V. Moise, Mecanisme (Mechanisms). (1999).

20. V. Valcovici, St. Balan, R. Voinea, Mecanică teoretică (Theoretical mechanics), (1963).

21. R. Voinea, D. Voiculescu, V. Ceauşu, Mecanică (Mechanics), (1985). 